\title{
Effect of Intervertebral Disk Degeneration on Spinal Stenosis During Magnetic Resonance Imaging With Axial Loading
}

\author{
Tae-Joon AHn, Sang-Ho LEE, Gun CHOI, Yong AHN, \\ Wei Chiang LIU*, Ho-Jin KIM, and Ho-Yeon LEE \\ Departments of Neurosurgery and * Radiology, Wooridul Spine Hospital, Seoul, R.O.K.
}

\begin{abstract}
Magnetic resonance (MR) imaging with axial loading can simulate the physiological standing state and disclose spinal stenosis undetected or underestimated in the conventional position. Intervertebral disk degeneration may be an important factor in spinal stenosis. This study investigated whether intervertebral disk degeneration increases spinal stenosis during axial loading. MR imaging with and without axial loading was obtained in 51 patients with neurogenic intermittent claudication and/or sciatica and reviewed retrospectively. The grade of disk degeneration was rated in four disk spaces from L2-3 to L5S1. The dural sac cross-sectional area (DCSA) was measured on MR images taken in both conventional and axial loading positions, and the change in the DCSA was calculated. The effect of disk degeneration on the DCSA was statistically analyzed. Significant decreases in the DCSA occurred with grade 4 disk degeneration (mean \pm standard deviation, $\left.20.1 \pm 14.1 \mathrm{~mm}^{2}\right)$, followed by grade $3\left(18.3 \pm 15.1 \mathrm{~mm}^{2}\right)$ and grade $2\left(8.9 \pm 13.1 \mathrm{~mm}^{2}\right)$. DCSA decreased considerably with increased severity of disk degeneration with axial loading, except for grade 5 disk degeneration. More accurate diagnosis of stenosis can be achieved using MR imaging with axial loading, especially if grade 2 to 4 disk degeneration is present.
\end{abstract}

Key words: magnetic resonance imaging, spinal stenosis, intervertebral disk

\section{Introduction}

Patients with spinal stenosis experience changes in their symptoms during certain activities or in different postures. The most common symptoms of lumbar spinal stenosis include neurogenic intermittent claudication (NIC), lower limb pain, numbness, paresthesia, and weakness. These symptoms are aggravated by standing, activity, and spinal extension, and relieved by sitting or spinal flexion. In general, lumbar flexion or a relaxed supine position with slight hip flexion will alleviate symptoms whereas standing or walking will provoke or aggravate symptoms. Forward flexion or a supine, psoasrelaxed position is well known to increase available space within the lumbar spinal canal, whereas walking or standing causes axial compression in the extension position that can provoke narrowing of the spinal canal.2,23,24) This dynamic phenomenon suggests that imaging techniques should be performed in the erect, extended position. ${ }^{19)}$

Received July 7, 2008; Accepted January 30, 2009
Magnetic resonance (MR) imaging of the spine is usually performed in a psoas-relaxed position, that is with the patient in the most comfortable supine position, with hips and knees flexed. Clearly, MR imaging in this position may not represent the state of the spinal canal during walking or standing, when the patient usually experiences symptoms, so carries considerable risk of underestimating or even missing stenosis of the spinal canal. Previously, MR imaging with axial loading, intended to achieve spinal alignment and loading similar to the standing position, has been recommended to simulate the lumbar spine in a standing state, in addition to conventional MR imaging. ${ }^{5,6,11,20,25,26)}$

Most humans older than 30 years show degenerative changes in the intervertebral disks. ${ }^{14)}$ Disk degeneration results in reduced hydration capacity and more fibrosis of the nucleus pulposus, leading to lower intradiscal pressure, ${ }^{21)}$ so the compressibility of the nucleus is increased during physiological axial loading state. ${ }^{7,13,18)}$ This may lead to increased redundancy of the discal, ligamentous, and meningeal tissues of the spine, resulting in increased cen- 
tral canal and lateral recess stenosis. ${ }^{10)}$

The present study evaluated the influence of intervertebral disk degeneration on central canal stenosis in the axial loaded state by comparing the dural sac cross-sectional area (DCSA) measured by MR imaging with and without axial loading.

\section{Materials and Methods}

Fifty-one patients, 27 women and 24 men aged 21-77 years (mean 51 years), with low back pain, NIC, and/or sciatica persisting for 2 weeks to 5 years (mean 11 months) who underwent MR imaging in the conventional psoas-relaxed and axial loading positions between July 2005 and February 2007 were retrospectively reviewed. None of the patients had prior history of lumbar surgery. Chief complaints were low back pain in one patient, sciatica in 36 patients, and NIC in 14 patients. A total of 157 disk levels were involved, including 20 L2-3 levels, 42 L3-4 levels, 50 L4-5 levels, and 45 L5-S1 levels. The Institutional Review Board approved the study.

MR imaging was performed with and without axial loading. All patients were first examined in a supine, psoas-relaxed position with a pillow under the knees to flex the hips and knees slightly (Fig. 1A), then with axial loading in the supine position with hips and knees extended (Fig. 1B). MR imaging was performed with a 1.5-T system (Magnetom Symphony; Siemens, Munchen, Germany) using a surface coil with repetition time/echo time $717 / 12 \mathrm{msec}$ for sagittal and axial $\mathrm{T}_{1}$-weighted images, 4500/132 msec for sagittal $\mathrm{T}_{2}$-weighted images, and 3700/120 msec for axial $\mathrm{T}_{2}$-weighted images. The image matrix was $512 \times 299$, field of view was $300 \times 300$ for sagittal images and $240 \times 240$ for axial images, the section thickness was $4 \mathrm{~mm}$, and the intersection gap was $0.2 \mathrm{~mm}$. The transverse MR imaging slices were oriented parallel to the endplate and at equal intervals as far as possible.

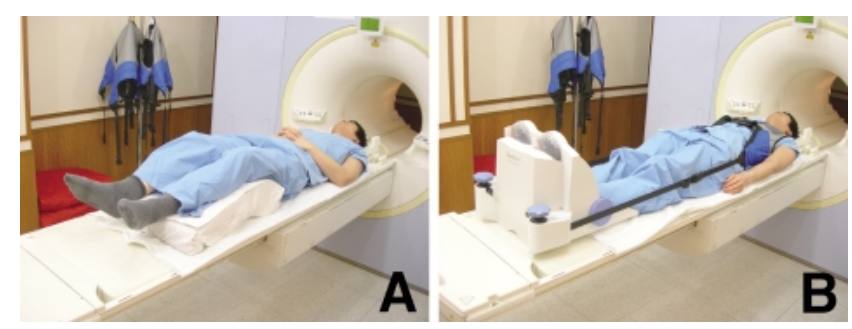

Fig. 1 Photographs illustrating use of the axial loading device during magnetic resonance imaging. A: Patient in the conventional position. B: Patient in the axial loading position.
Axial loading was applied with a commercially available device (Dyna Well L-spine; Dyna Well Int. AB, Billdal, Sweden), approved by the United States Food and Drug Administration, to simulate the lumbar spine in an upright, axial loaded position (Fig. 1B). This device consists of a harness/jacket with straps connected to a footplate and a compression device. The patient wears the harness/jacket and lies in the supine position. The feet are placed against the footplate and the axial load is applied through the side straps by turning the adjustment knobs. Approximately $50 \%$ of the patient's body weight is applied, distributed equally between the legs. ${ }^{5,9,11,16,21,26)}$ The load was applied for at least 5 minutes $^{5,9)}$ and then MR imaging was performed using the same protocols as in the conventional position. During the examination, the patients were asked regularly about pain in the back or legs.

The DCSA was measured at 4 disk levels between L2-3 and L5-S1 on the images selected in which the DCSA seemed to be the smallest at each disk and lateral recess level. ${ }^{25)}$ The most comparable MR images taken in the conventional and axial loading positions were selected by careful inspection of nerve roots, surrounding soft tissues, and bony structures such as facet joints and lamina ${ }^{5,26)}$ by a neurosurgeon and a neuroradiologist. The DCSA was determined on axial $\mathrm{T}_{2}$-weighted images using the standard measurement tools provided in the software (PiView $^{\mathrm{TM}}$; INFINITT Co., Ltd., Seoul, R.O.K.) (Fig. 2). The DCSA was measured three times on each image separately at intervals of several weeks, and the mean value was used to reduce measurement errors. ${ }^{5,6)}$ Changes in the DCSA between the conventional and axial loading positions were calculated.

Disk degeneration was also assessed on midsagittal $\mathrm{T}_{2}$-weighted images according to the proposed grading system. ${ }^{17)}$ Grade 1 is a homogeneous, bright and white-looking disk, grade 2 is an inhomogeneous disk with or without horizontal bands, grade 3 is a gray disk with unclear distinction of the nucleus and annulus, grade 4 is a gray to black disk with no distinction of the nucleus and annulus, and grade 5 is a black disk with collapsed disk space. Disk degeneration was graded separately by a neurosurgeon and a neuroradiologist. Both observers rated the grade of disk degeneration twice with an interval of several weeks. The intra-observer and inter-observer reliability was evaluated.

The effect of disk degeneration on the DCSA change between conventional and axial loading positions was evaluated. Data were analyzed with a software program (SAS version 8.1; SAS Institute Inc., Cary, N.C., U.S.A.). Change in the DCSA be- 

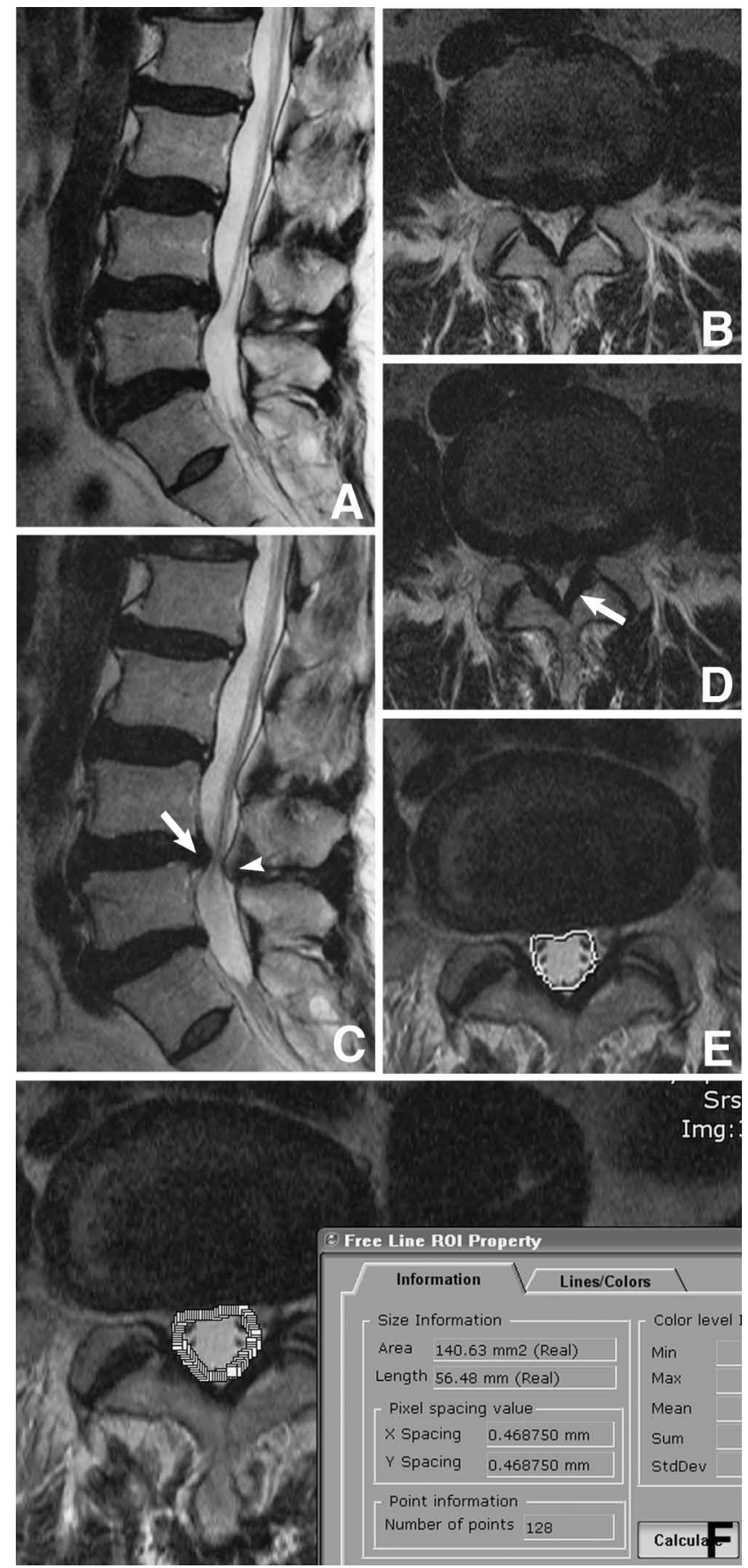

Fig. 2 Magnetic resonance images taken in the conventional (A, B) and axial loading positions (C, D) showing more collapsed disk space and more herniated disk (C, arrow), buckling of the ligamentum flavum (C, arrowhead), and smaller dural sac cross-sectional area (DCSA) (D, arrow) with axial loading. Measurement of the DCSA on an axial $\mathbf{T}_{2}$-weighted magnetic resonance image (E), and software calculation and display of the DCSA (F). tween conventional and axial loading positions was evaluated within the same grade of disk degeneration using the paired t-test and the Wilcoxon signed rank test. Change in the DCSA between the grades of disk degeneration was evaluated using the analysis of variance test and two sample t-tests. Intra-observer and inter-observer reliability were investigated using the kappa test. A p value of less than 0.05 was considered statistically significant.

\section{Results}

Evaluation of disk degeneration at 157 disk levels in the 51 patients identified $1(0.6 \%)$ disk with grade 1 , $29(18.5 \%)$ disks with grade 2, 48 (30.6\%) disks with grade 3, 68 (43.3\%) disks with grade 4, and 11 (7.0\%) disks with grade 5 . Assessment of inter-observer reliability ( $\kappa=0.86, \kappa=0.87$ ) and intra-observer reliability $(\kappa=0.84)$ demonstrated excellent agreement (kappa values between 0.81 and 1.00 indicate excellent agreement ${ }^{12)}$ ). There was only one disk with grade 1 degeneration, so statistical analysis was performed for grade 2, 3, 4, and 5 disk degeneration.

The findings of DCSA change are given in Table 1. DCSA decrease under axial loading most frequently occurred with grade 4 disk degeneration $(89.7 \%)$, followed by grade $3(85.4 \%)$. DCSA showed significant differences between conventional and axial loading positions with grade 2,3 , and 4 disk degeneration $(p<0.01)$, but not with grade $5(p=$ 0.14). The most significant decrease in DCSA occurred with grade 4 (mean \pm standard deviation, $\left.20.1 \pm 14.1 \mathrm{~mm}^{2}\right)$, followed by grade $3(18.3 \pm 15.1$ $\left.\mathrm{mm}^{2}\right)$ and grade $2\left(8.9 \pm 13.1 \mathrm{~mm}^{2}\right)$. Figure 3 shows percentage of DCSA reduction after axial loading. Comparison of DCSA in groups with different grades of disk degeneration showed statistically significant differences between grades 2 and $3(\mathrm{p}=$ $0.01)$, grades 2 and $4(\mathrm{p}<0.01)$, grades 3 and $5(\mathrm{p}=$ $0.01)$, and grades 4 and $5(\mathrm{p}<0.01)$.

\section{Discussion}

Many authors have reported the usefulness of MR imaging with axial loading using a compressive device. MR imaging with axial loading can disclose pathological features including central spinal stenosis not detected by conventional MR imaging. For example, 29 of 34 patients demonstrated significant DCSA reduction, to lower than $100 \mathrm{~mm}^{2}{ }^{26)}$ Similar results were also reported. ${ }^{6)}$ Lumbar DCSA below 75 $\mathrm{mm}^{2}$ indicates absolute central stenosis, below 100 $\mathrm{mm}^{2}$ indicates relative central stenosis, and between 100 and $130 \mathrm{~mm}^{2}$ indicates early stenosis. ${ }^{3,8,22,26)}$ The reduction in DCSA between MR imaging in the con- 
Table 1 Measurement of dural sac cross-sectional area (DCSA) by magnetic resonance imaging

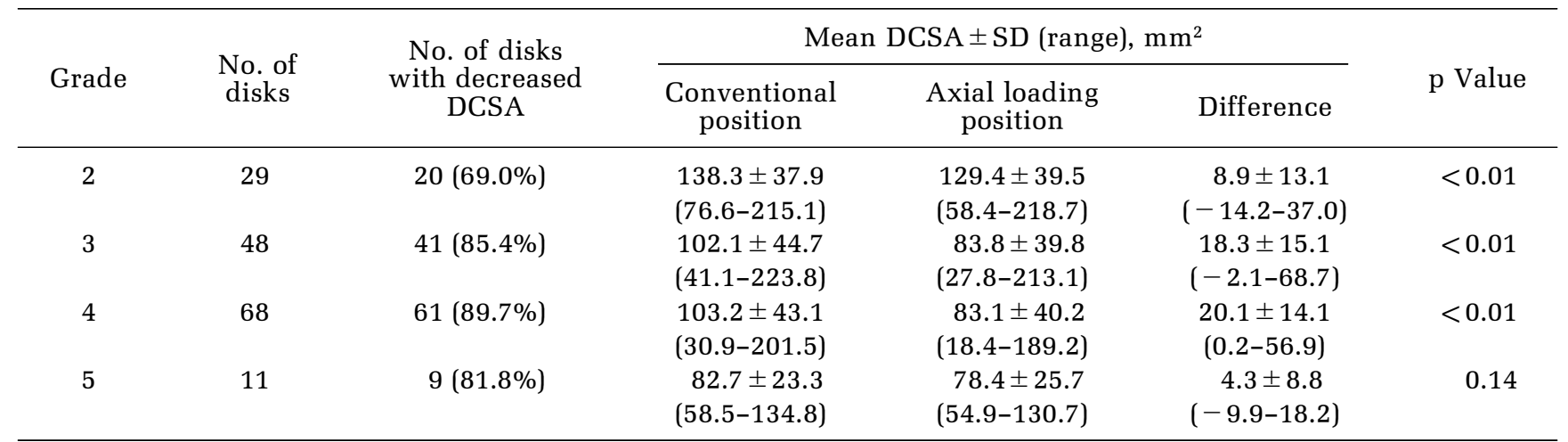

SD: standard deviation.

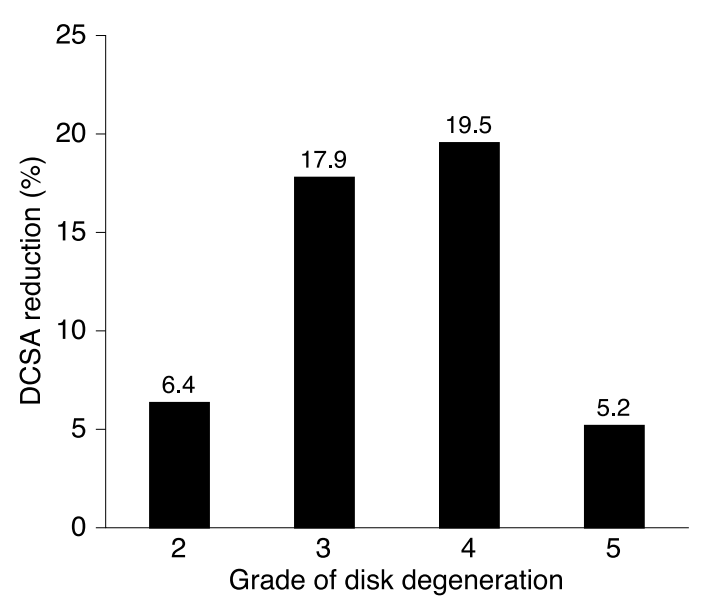

Fig. 3 Percentage of dural sac cross-sectional area (DCSA) reduction after axial loading. DCSA decreased more with axial loading except for grade 5 disk degeneration, as the disk is more degenerated.

ventional and axial loading positions was more pronounced in older subjects than in asymptomatic, young subjects. ${ }^{5)}$

Occult nerve root compression could be identified by MR imaging with axial loading, but not with conventional MR imaging in the supine position. ${ }^{20)} \mathrm{MR}$ imaging with axial loading provides additional information, including deformation of the dural sac, increased disk protrusion, and visualization of synovial cyst. ${ }^{9)}$ Such information may encourage experienced neurosurgeons to change treatment plans in significant numbers of patients. However, MR imaging with axial loading should be avoided in patients with vertebral fracture, severe osteoporosis, or other pathological conditions, so all patients should be first evaluated by MR imaging in the conventional position. ${ }^{5,25)}$ Some patients complain of se- vere back pain or sciatic pain during axial loading. In our institution, axial loaded MR imaging could not be completed in about $10 \%$ of patients due to back pain or sciatic pain.

Measurement of the DCSA is the most accurate method to determine central lumbar stenosis. ${ }^{3)}$ Many authors have used the DCSA as a standard diagnostic method to estimate central canal stenosis. $4,6,8,19,26)$ Comparison of measurements of the anteroposterior diameter of the bony canal by computed tomography, the anteroposterior diameter of the dural sac by myelography, and the cross-sectional area of the dural sac by computed tomography with surgical findings in 24 patients with a clinical diagnosis of central canal stenosis revealed that DCSA measurement was the most accurate and effective method to diagnose central canal stenosis. ${ }^{3)}$

Disk degeneration is associated with reduced hydration capacity of the nucleus pulposus with more fibrotic appearance and less effective resistance to compressive forces in the spine, ${ }^{15,18)}$ leading to decreased disk height and less taut annulus fibrosus, with consequent diffuse disk bulging and inward buckling of the ligamentum flavum. ${ }^{19,27)}$ In vitro studies have shown that degenerated disks have less water content and reduced volume of the nucleus pulposus, resulting in decreased intradiscal pressure and increased compressibility of the nucleus. ${ }^{1)}$ In addition, the laxity of the anterior and posterior longitudinal ligaments allows greater tensile deformation of the disk in a degenerated disk. Intradiscal pressure measurements in normal and degenerated disks found reduced intradiscal pressure with increasing degeneration. ${ }^{18,21)}$ The height of intervertebral disk is also reduced with increasing disk degeneration, which causes decreased forces in the ligamentum flavum and posterior longitudinal ligament, and eventually ligament buckling. ${ }^{18)}$ With disk degeneration, the spine may collapse into itself 
under physiological axial loading, which is termed spinal column telescoping, leading to increased redundancy of the disk, ligaments, and meningeal tissues of the spine. ${ }^{10)}$

Spinal stenosis has many etiologies including disk herniation, ligamentum flavum thickening or buckling, facet joint hypertrophy, and even facet joint cyst, but this study focused on the severity of disk degeneration and axial loading of the spine. The decrease of DCSA measured by MR imaging in the conventional and axial loading positions was statistically significant at grades 2,3 , and 4 . Grade 5 disk degeneration corresponds to collapse of the disk space, so the axial loading position may not have clear advantages over the conventional position. However, increased severity of disk degeneration from grades 2 to 4 was reflected in decreased DCSA under axial loading (Fig. 2).

This study included only one case of grade 1 degeneration, so comparing normal with degenerated disks was not possible. Most MR images were obtained in middle to older aged individuals with lumbar problems, so few normal disks were observed. Investigation of asymptomatic, young individuals may be helpful.

Analysis of 157 disk levels in 51 patients demonstrated that the DCSA decreased considerably with increased severity of disk degeneration as measured by comparing MR imaging in the axial loading and conventional positions. Grade 5 disk degeneration demonstrated no difference. More accurate diagnosis of spinal stenosis could be achieved with axial loading, especially if advanced disk degeneration is present, except for grade 5 disk degeneration.

\section{Acknowledgment}

This study was supported by a grant from Wooridul Spine Foundation.

\section{References}

1) Adams MA, Bogduk N, Burton K, Dolan P: The Biomechanics of Back Pain. Edinburgh, London, New York, Churchill Livingstone, 2002, 100 pp

2) Amundsen T, Weber H, Lilleas F, Nordal HJ, Abdelnoor M, Magnaes B: Lumbar spinal stenosis. Clinical and radiologic features. Spine 20: 1178-1186, 1995

3) Bolender NF, Schonstrom NS, Spengler DM: Role of computed tomography and myelography in the diagnosis of central spinal stenosis. J Bone Joint Surg Am 67: 240-246, 1985

4) Coulier B, Devyver B, Ghosez JP: Severe underestimation of lumbar spinal stenosis by supine imaging. Clin Radiol 58: 167-169, 2003

5) Danielson B, Willen J: Axially loaded magnetic resonance image of the lumbar spine in asymptomatic individuals. Spine 26: 2601-2606, 2001

6) Danielson BI, Willen J, Gaulitz A, Niklason T, Hansson TH: Axial loading of the spine during CT and MR in patients with suspected lumbar spinal stenosis. Acta Radiol 39: 604-611, 1998

7) Gunzburg R, Parkinson R, Moore R, Cantraine F, Hutton W, Vernon-Roberts B, Fraser R: A cadaveric study comparing discography, magnetic resonance imaging, histology, and mechanical behavior of the human lumbar disc. Spine 17: 417-426, 1992

8) Hamanishi C, Matukura N, Fujita M, Tomihara M, Tanaka S: Cross-sectional area of the stenotic lumbar dural tube measured from the transverse views of magnetic resonance imaging. J Spinal Disord 7: 388-393, 1994

9) Hiwatashi A, Danielson B, Moritani T, Bakos RS, Rodenhause TG, Pilcher WH, Westesson PL: Axial loading during MR imaging can influence treatment decision for symptomatic spinal stenosis. AJNR Am J Neuroradiol 25: 170-174, 2004

10) Jinkins JR, Dworkin JS, Damadian RV: Upright, weight-bearing, dynamic-kinetic MRI of the spine: initial results. Eur Radiol 15: 1815-1825, 2005

11) Kimura S, Steinbach GC, Watenpaugh DE, Hargens AR: Lumbar spine disc height and curvature responses to an axial load generated by a compression device compatible with magnetic resonance imaging. Spine 26: 2596-2600, 2001

12) Landis JR, Koch GG: The measurement of observer agreement for categorical data. Biometrics 33: 159-174, 1977

13) McNally DS, Adams MA: Internal intervertebral disc mechanics as revealed by stress profilometry. Spine 17: 66-73, 1992

14) Miller JA, Schmatz C, Schultz AB: Lumbar disc degeneration: correlation with age, sex, and spine level in 600 autopsy specimens. Spine 13: 173-178, 1988

15) Mimura M, Panjabi MM, Oxland TR, Crisco JJ, Yamamoto I, Vasavada A: Disc degeneration affects the multidirectional flexibility of the lumbar spine. Spine 19: 1371-1380, 1994

16) Nachemson AL: Disc pressure measurements. Spine 6: 93-97, 1981

17) Pfirrmann CW, Metzdorf A, Zanetti M, Hodler J, Boos N: Magnetic resonance classification of lumbar intervertebral disc degeneration. Spine 26: 1873-1878, 2001

18) Rohlmann A, Zander T, Schmidt H, Wilke HJ, Bergmann G: Analysis of the influence of disc degeneration on the mechanical behaviour of a lumbar motion segment using the finite element method. J Biomech 39: 2484-2490, 2006

19) Saifuddin A: The imaging of lumbar spinal stenosis. Clin Radiol 55: 581-594, 2000

20) Saifuddin A, Blease S, MacSweeney E: Axial loaded MRI of the lumbar spine. Clin Radiol 58: 661-671, 2003

21) Sato K, Kikuchi S, Yonezawa T: In vivo intradiscal 
pressure measurement in healthy individuals and in patients with ongoing back problems. Spine 24: 2468-2474, 1999

22) Schonstrom N, Hansson T: Pressure changes following constriction of the cauda equina. An experimental study in situ. Spine 13: 385-388, 1988

23) Schonstrom N, Lindahl S, Willen J, Hansson T: Dynamic changes in the dimensions of the lumbar spinal canal: an experimental study in vitro. J Orthop Res 7: 115-121, 1989

24) Sortland O, Magnaes B, Hauge T: Functional myelography with metrizamide in the diagnosis of lumbar spinal stenosis. Acta Radiol Suppl 355: 42-54, 1977

25) Willen J, Danielson B: The diagnostic effect from axial loading of the lumbar spine during computed tomography and magnetic resonance imaging in patients with degenerative disorders. Spine 26: 2607-2614, 2001

26) Willen J, Danielson B, Gaulitz A, Niklason T, Schonstrom N, Hansson T: Dynamic effects on the lumbar spinal canal: axially loaded CT-myelography and MRI in patients with sciatica and/or neurogenic claudication. Spine 22: 2968-2976, 1997

27) Zamani AA, Moriarty T, Hsu L, Winalski CS, Schaffer JL, Isbister $\mathrm{H}$, Schenck JF, Rohling KW, Jolesz F: Functional MRI of the lumbar spine in erect position in a superconducting open-configuration MR system: preliminary results. J Magn Reson Imaging 8: 1329-1333, 1998

Address reprint requests to: Yong Ahn, M.D., Department of Neurosurgery, Wooridul Spine Hospital, 47-4 Chungdam-dong, Gangnam-gu, Seoul, 135-100 R.O.K.

e-mail:ns-ay@hanmail.net

\section{Commentary}

The authors correlate the degree of disk degeneration with the increase of dural sac compression, as observed by MRI using axial loading. They demonstrate that there is a clear and significant correlation between grades 2 to 4 MRI disk change and an increased effect of axial loading on useful spinal canal cross section. Not surprisingly, a complete disk collapse (grade 5) does not allow any further modification under axial loading. The results, although not new, are well documented and clearly presented. I think that any neurosurgeon dealing with spinal problems should keep in mind this possibility. It would be interesting to compare these data with those obtained by MRI carried out in the standing position, that has been recently introduced in clinical practice.

Alessandro DuCATI, M.D. Ordinario di Neurochirurgia Universita' di Torino Torino, Italy

Over the last few years, we have had access to an MRI scanner where we could do both lying and standing pictures. We have similar data to that reported by Dr. Ahn et al. But the Wooridul Spine Hospital has done a superb job in classifying all of this. Everyone needs to look at Figure 2 and compare the pictures of $A$ and $B$ to $C$ and $D$. Then the next thing they need to do is look at Figure 3, because it is in the grade III and grade IV disks where this is quite obvious. The ones that are in grade II still have enough elasticity not to be compressed. The grade $V$ disk degenerations are already collapsed and stiff and they are not going to move much. With a good patient history of neurogenic claudication, MRI pictures with grade III and grade IV disks, you can be well assured that a compression or standing MRI will show the stenosis considerably worse. We use such MRIs to show patients why on standing and walking they become so symptomatic. We allow these patients to make their own decisions when and if they need surgery. But once you explain the process, show them the pictures and outline the basic surgical procedure, by far the majority of the patients want their cauda equina decompressed.

Thomas B. DuCKER, M.D., F.A.C.S. Professor of Neurosurgery Maryland Brain \& Spine, LLC Annapolis, Maryland, U.S.A. 